\title{
Laser-driven high-energy proton beam with homogeneous spatial profile from a nanosphere target
}

\author{
D. Margarone, ${ }^{1}$ I. J. Kim, ${ }^{2,3}$ J. Psikal, ${ }^{1,4}$ J. Kaufman, ${ }^{1,4}$ T. Mocek, ${ }^{5}$ I. W. Choi, ${ }^{2,3}$ L. Stolcova, ${ }^{1,4}$ \\ J. Proska, ${ }^{4}$ A. Choukourov, ${ }^{1,6}$ I. Melnichuk, ${ }^{6}$ O. Klimo, ${ }^{1,4}$ J. Limpouch, ${ }^{1,4}$ J. H. Sung, ${ }^{2,3}$ \\ S. K. Lee, ${ }^{2,3}$ G. Korn, ${ }^{1}$ and T. M. Jeong ${ }^{2,3, *}$ \\ ${ }^{1}$ ELI-Beamlines Project, Institute of Physics ASCR, v.v.i. (FZU), 18221 Prague, Czech Republic \\ ${ }^{2}$ Center for Relativistic Laser Science, Institute for Basic Science, Gwangju 500-712, Republic of Korea \\ ${ }^{3}$ Advanced Photonics Research Institute, GIST, Gwangju 500-712, Republic of Korea \\ ${ }^{4}$ FNSPE, Czech Technical University in Prague, 11519 Prague, Czech Republic \\ ${ }^{5}$ HiLASE project, Institute of Physics of the ASCR, 18221 Prague, Czech Republic \\ ${ }^{6}$ Faculty of Mathematics and Physics, Department of Macromolecular Physics, \\ Charles University in Prague, Czech Republic
}

(Received 11 February 2015; published 10 July 2015)

\begin{abstract}
A high-energy, high-yield proton beam with a good homogeneous profile has been generated from a nanosphere target irradiated by a short (30-fs), intense $\left(7 \times 10^{20} \mathrm{~W} / \mathrm{cm}^{2}\right)$ laser pulse. A maximum proton energy of $30 \mathrm{MeV}$ has been observed with a high proton number of $7 \times 10^{10}$ in the energy range $5-30 \mathrm{MeV}$. A homogeneous spatial profile with a uniformity (standard deviation from an average value within $85 \%$ beam area) of $15 \%$ is observed with the nanosphere dielectric target. Particle-in-cell simulations show the enhancement of proton cutoff energy and proton number with the nanosphere target and reveal that the homogeneous beam profile is related with a broadened angular distribution of hot electrons, which is initiated by the nanosphere structure. The homogeneous spatial properties obtained with the nanosphere target will be advantageous in developing laser-driven proton sources for practical applications in which high-quality beams are required.
\end{abstract}

DOI: 10.1103/PhysRevSTAB.18.071304

PACS numbers: 52.38.Ph, 41.75.Jv, 52.50.Dg

\section{INTRODUCTION}

In the past decade, great attention has been paid to laser-driven ion acceleration as an innovative approach for medical applications, especially cancer therapy [1]. Because of the novel concept, research groups have mostly focused on the basic performances of laser-accelerated ion beams, i.e., the increase in the maximum proton or ion energy using advanced targets, the generation of quasimonoenergetic energy spectra, and the search for efficient acceleration mechanisms. As a result, the production of 60-70-MeV protons [2], quasimonoenergetic features in the spectrum $[3,4]$, and various acceleration schemes, such as target normal sheath acceleration (TNSA) [5], radiationpressure acceleration (RPA) [6,7], break-out afterburner $[8,9]$, and hybrid TNSA-RPA $[10,11]$, were reported in the literature. Despite the fast progress and great potential of laser-driven ion acceleration, a number of issues including the increase of proton energy up to a few hundreds of $\mathrm{MeV}$, high-conversion efficiency, reduced shot-to-shot

\footnotetext{
"To whom all correspondence should be addressed. jeongtm@gist.ac.kr

Published by the American Physical Society under the terms of the Creative Commons Attribution 3.0 License. Further distribution of this work must maintain attribution to the author $(s)$ and the published article's title, journal citation, and DOI.
}

fluctuations in the energy spectrum, and improved ion beam quality are still considered as main topics to be investigated and resolved in the near future [12].

The use of laser-driven proton sources in practical applications requires the production of reliable, highenergy, and high-conversion-efficiency proton beams. As an effort in this direction, a micron-thick plastic foil target covered by a nanosphere monolayer was proposed [13], and its capability of producing $\sim 10-\mathrm{MeV}$ proton beams with enhanced conversion efficiency was experimentally demonstrated by using a 100-TW-class laser at an intensity of $5 \times 10^{19} \mathrm{~W} / \mathrm{cm}^{2}$ [14]. The role of the nanosphere monolayer was to boost the laser absorption on the laser-irradiated target surface. The higher absorption enhanced the hot electron population generated. As a consequence, the proton beam cutoff energy was increased by a factor of 1.5 and the number of protons by a factor of 6 . The main motivation of our work is to extend the validity of such an acceleration mechanism at higher laser intensities and also to report the improved homogeneity of the proton beam spatial profile in order to show its potential use when higher proton energies (100-MeV level), which are needed for medical applications (namely, hadron therapy), are obtained.

In this paper, we report on laser-driven proton acceleration using nanosphere targets (similar to the previous ones in Refs. [14] and [15]) at higher laser intensities (up to 
$7 \times 10^{20} \mathrm{~W} / \mathrm{cm}^{2}$ ) confirming the enhancement of the proton cutoff energy and the increase of the proton number. The completely new achievement is represented by the generation of a homogeneous proton beam spatial profile, which is experimentally observed and considered as an additional benefit of the use of a nanosphere insulator target. In fact, irregular proton beam spatial profiles are common for a dielectric target, and their origin can be explained by several mechanisms [16]. Our two-dimensional (2D) particle-in-cell (PIC) simulations show good agreement with the experimental observations in terms of proton cutoff energy and proton number and also provide an interpretation of the origin of the homogeneous beam spatial profile ascribable to a broad angular distribution of hot electrons on the front surface of the nanosphere target. Both experimental and numerical results show that a micron-thick foil target with a nanosphere monolayer has a crucial importance of producing high-energy high-quality proton beams through laser-matter interaction at higher laser intensities; thus, the nanosphere target can be used as a proton beam source for multidisciplinary applications.

\section{EXPERIMENTAL SETUP AND DIAGNOSTICS}

Our acceleration experiments were performed with the 30-fs, 0.1-Hz-repetition-rate, 1-PW laser operating at the Center for Relativistic Laser Science (CoReLS), Institute for Basic Science (IBS) in Korea. The overall output performance of the petawatt laser is described elsewhere [17]. A double plasma mirror system was employed to maintain the temporal contrast ratio (ratio between the intensity of a main femtosecond pulse and the intensity of a nanosecond pedestal) as low as $3 \times 10^{11}$ up to 6 ps before the main laser pulse. This is a crucial parameter when using a target with special structures on the front surface which should be sustained from the preplasma formed by amplified spontaneous emission and prepulse before the main laser pulse [18]. On the other hand, the laser energy on target was reduced by a factor of more than 2 compared to the output at the exit of the pulse compressor chamber, due to the presence of a double plasma mirror system which was used to enhance the laser pulse temporal contrast ratio on target. The laser pulse was focused with an $f / 3$ off-axis parabolic (OAP) mirror, and the measured spot size was $\sim 4 \mu \mathrm{m}$ at FWHM, yielding a maximum peak intensity of $7 \times 10^{20} \mathrm{~W} / \mathrm{cm}^{2}$. Such an intensity value was estimated by considering a laser pulse energy of $\sim 30 \mathrm{~J}$ at the exit of the pulse compressor reduced to $\sim 10 \mathrm{~J}$ delivered on target because of the upstream optical elements (double plasma mirror, large-aperture half-wave plates, and gold-coated OAP). Furthermore, a laser energy of $2.5 \mathrm{~J}$ (25\%) out of $10 \mathrm{~J}$ was contained in the $4-\mu \mathrm{m}$ focal spot. The laser beam was $s$-polarized on target.

Two types of targets were used in the experiment: (i) planar target (PT) consisting of a 700-nm-thick Mylar foil and (ii) nanosphere target (NST) with an effective thickness of $720 \mathrm{~nm}$. The latter consisted of a monolayer of polystyrene spheres $(470 \mathrm{~nm}$ in diameter) placed on a double layer substrate (200-nm-thick silicon nitride membrane and 50-nm-thick polyethylene film on the rear side). Details on the fabrication of target substrates (hydrocarbon polymeric thin films) using the physical vapor deposition technique can be found in Ref. [19], and the fabrication of the nanosphere target is based on the self-assembly at the water-air interface as described in Ref. [20]. The damagethreshold fluence of the nanosphere structure has been estimated to range between 1 and $10 \mathrm{~J} / \mathrm{cm}^{2}$ for approximate nanosecond laser pulses [21]; thus, the nanosphere structure was operational in our experimental conditions, in which the prepulse intensity was maintained at $10^{9}-10^{10} \mathrm{~W} / \mathrm{cm}^{2}$ when considering the temporal contrast ratio. The target was inclined by $9^{\circ}$ to the laser propagation direction to avoid the laser pulse retroreflection. The proton and ion energy spectra were measured by a Thomson Parabola (TP) spectrometer equipped with a microchannel plate (MCP) and a 16-bit charge-coupled device (CCD). A phosphor screen in the MCP was placed $12 \mathrm{~cm}$ away from the output plane of the electric field $(25.5 \mathrm{kV} / \mathrm{cm})$ and the magnetic field gradient $(0.4 \mathrm{~T} / \mathrm{cm})$ deflection regions. The image on the phosphor screen was relayed to the CCD camera for a real-time measurement. A typical TP
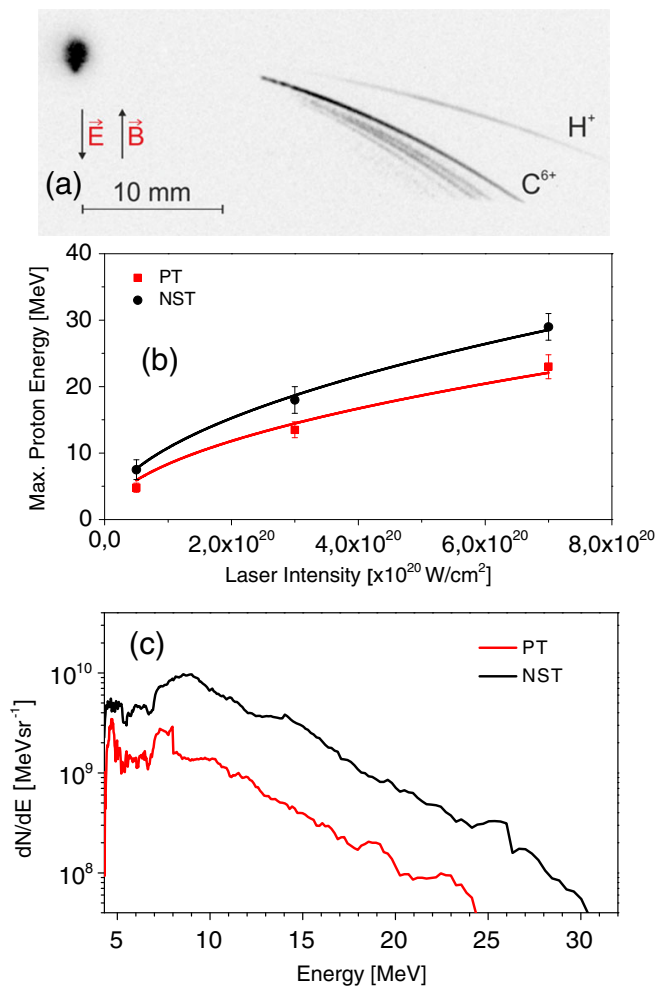

FIG. 1. Typical TP snapshot for a NST target (a); maximum proton energy vs laser intensity in three different experimental campaigns at CoReLS-IBS (b); typical proton energy distributions for PT and NST targets at $7 \times 10^{20} \mathrm{~W} \mathrm{~cm}^{-2}$ (c). 
spectrum is shown in Fig. 1(a). The absolute number calibration for the proton energy spectra was done by installing striped CR-39 track detectors in front of the MCP device [22]. A stack of radiochromic film (RCF) detectors was placed $5 \mathrm{~cm}$ behind the target mainly for an ex situ estimation of the entire proton beam spatial profile and divergence in different proton energy ranges [23]. The parasitic dose contribution by high-energy protons in a certain RCF layer was corrected by a post-data-analysis process using predetermined weighting coefficients obtained from the proton Bragg curves simulated in the stopping and range of ions in matter.

\section{RESULTS AND DISCUSSIONS}

As demonstrated in previous papers [14,15], both the proton cutoff energy and the number of protons further increased with nanosphere targets at higher intensities. Figure 1(b) shows the proton cutoff energy as a function of laser intensity on the target. The experimental data referring to three different campaigns at different laser intensities of about $5 \times 10^{19}, 3 \times 10^{20}$, and $7 \times 10^{20} \mathrm{~W} / \mathrm{cm}^{2}$, respectively, are summarized. The maximum proton energy roughly scales with the square root of the laser intensity, which is considered as the typical scaling law in the TNSA regime. The maximum proton energy for the nanosphere target is roughly 1.5 times higher than for the planar one in the whole laser intensity range.

Figure 1(c) shows proton energy spectra for PT and NST targets recorded with the TP spectrometer. The maximum cutoff energies (with a corresponding intensity 5 times higher than the noise standard deviation after background subtraction) for PT and NST targets are about 24 and $30 \mathrm{MeV}$, respectively. Thus, an increase of about $25 \%$ in the maximum proton energy is achieved with the NST target. The total proton yield for energies above $5 \mathrm{MeV}$ is also enhanced from $1.5 \times 10^{10}$ for PT to $6.9 \times 10^{10}$ for NST, resulting in an increase of $350 \%$ (a factor of about 4.5 ) in the total proton number. If the proton number in the energy window 13-23 MeV is considered, the enhancement is more than a factor of 3 for NST compared to PT. The proton acceleration efficiency, defined by the ratio between the total proton beam energy [integral of $E \cdot f(E)$, where $E$ is the proton energy and $f(E)$ is the proton energy distribution] and the laser energy, is also enhanced by a factor of about 5 (from $0.3 \%$ for planar targets to $1.5 \%$ for nanosphere targets).

The enhancement of the proton cutoff energy and the proton number are also confirmed by PIC simulations. The 2D PIC code described in Ref. [24] was employed for detailed analyses of experimental results. The input parameters of simulation runs were appropriately adjusted to circumvent the limitation of the 2D PIC method. In the simulations, both the planar foil and the nanosphere target consist of $\mathrm{C}^{6+} \mathrm{H}_{2}{ }^{+}$plasma with an electron density $\left(\mathrm{n}_{\mathrm{e}}\right)$ of $3.5 \times 10^{23} \mathrm{~cm}^{-3}\left(200 \mathrm{n}_{\mathrm{c}}\right)$, where $\mathrm{n}_{\mathrm{c}}$ is the critical electron density. The planar foil target has a thickness of $750 \mathrm{~nm}$. The nanosphere target consists of a monolayer of closely packed nanospheres on the front (laserirradiated) side and a 250-nm-thick dielectric substrate similarly to the real geometry measured through SEM analyses [20]. Each nanosphere has a diameter of $460 \mathrm{~nm}$. The beam width of the focal spot is set to $1.6 \mu \mathrm{m}$ (at FWHM). Gaussian distributions in temporal and spatial profiles are assumed in simulations. A smaller beam diameter than the experimental one is used to represent 3D-lateral losses of hot electrons in the 2D-geometry model as discussed in Ref. [14]. The target is initialized with a steplike density profile. However, a 30 -fs prepulse with one-hundredth peak intensity of the main pulse, which arrives 50 fs prior to the main pulse interaction (based on spectral phase interferometry for direct electric-field reconstruction measurements) and creating a short preplasma on the target, is introduced in our simulations. Since the diameter of the spheres is several times smaller than the focal spot size, the result is not sensitive to the distribution of the nanospheres owing to the laser focal spot position. In a real 3D geometry, the electrons traveling on a line parallel to the surface would experience a spatial periodicity equal to the size of the sphere only along a limited set of directions [25]. According to their initial acceleration direction, electrons can cross any vacuum region between any couple of spheres or can travel along a different path, showing an even more complicated periodicity of the system. However, as observed in our first theoretical proposal [13], the results are not very different when a monolayer of nanospheres having random diameters in the range 200-800 $\mathrm{nm}$ are used compared to nanospheres having a fixed diameter. Thus, even if multidimensional effects are more complicated, we expect a similar qualitative difference between the nanosphere target and the flat foil when comparing 2D and 3D PIC simulation outputs.

The simulation results for proton energy spectra are shown in Fig. 2. The energies of accelerated protons at the end of the simulations (200 fs after laser-target interaction)

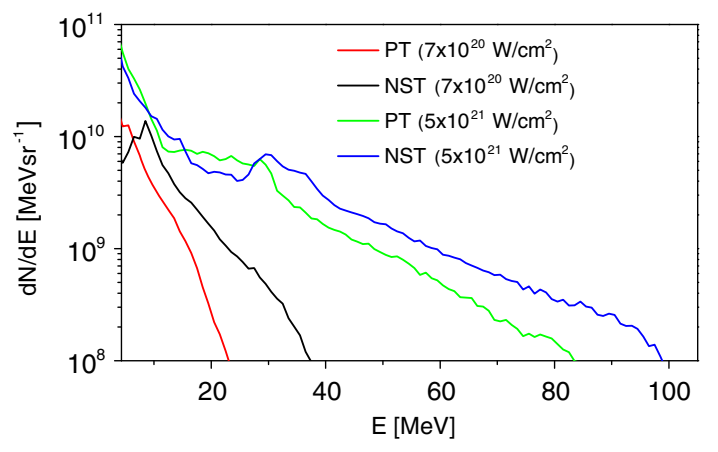

FIG. 2. Proton energy spectra for planar and nanosphere targets at the end of PIC simulations. 
were compared with the experimental ones. The cutoff energy agrees relatively well for a planar target, whereas the proton energy from a nanosphere target is overestimated in the PIC simulation. The higher cutoff energy in the PIC simulation $(\sim 23 \%)$ can be explained by larger lateral losses of hot electrons in the experiment (two lateral dimensions in 3D) due to their larger angular spread at the target front surface. As already mentioned, only one lateral dimensional loss is considered in our 2D PIC simulations, and we applied a correction factor based on the decrease of the laser beam size in the 2D simulations. However, such a correction is fully applicable only for planar foils, since in the case of nanosphere targets a focal spot size below $1 \mu \mathrm{m}$ (close to the diffraction limit) should have been used. This was not possible to be set up in our PIC simulations. Therefore, we applied the same correction to planar and nanosphere targets. The envisioned proton energy spectrum with the same nanosphere target at even higher laser intensity is also plotted in Fig. 2. According to the PIC simulations, a cutoff energy of $100 \mathrm{MeV}$ is expected when a nanosphere target is irradiated by a laser pulse with an intensity of $5 \times 10^{21} \mathrm{~W} / \mathrm{cm}^{2}$ that is still in agreement with the square root of the laser intensity scaling law valid in the TNSA regime.

Figure 3 shows proton beam profiles recorded with RCF layers at different proton energies. The images in Fig. 3 were obtained by scanning RCFs with three shots accumulated for the NST and five shots for the PT, and the image quality was improved with a linear pseudocolor scale. As for the PT, the proton beam divergence decreases from $\sim 30^{\circ}$ to $\sim 20^{\circ}$ as the proton energy increases. A significant decrease to $\sim 6^{\circ}$ in the divergence angle was observed in a RCF layer located close to the cutoff energy. The same result can be observed in PIC simulations. As for the NST, the divergence angle remains constant for all proton energies $\left(\sim 25^{\circ}\right)$. Moreover, a ring structure is observed outside the proton beam profile. Such a ring structure is very regular and thus can be easily removed with a proper collimator. It is inferred from PIC simulations that a higher divergence of protons at a higher energy is related with the lateral expansion of outermost peak structures (corresponding to a ring structure in the experiment) in the angular distribution of protons (as discussed below).
Figures 4(a) and 4(b) show the angular spectrum and the momentum of hot electrons in the phase space for the planar and the nanosphere targets from PIC simulations. The difference in angular distribution can be explained by trajectories of electrons on the front surface of the targets as shown in Ref. [25], and it agrees well with our previous numerical results obtained at a lower laser intensity [13]. Hot electrons can be generated when the electric field associated with the laser pulse, which forces electrons to oscillate in vacuum, is shielded by the plasma (ionized target). The shielding of the laser pulse electric field strongly depends on the morphology of the target front surface. When this surface consists of multiple curvatures, such as in the case of a nanosphere target, the electric field can be shielded in various directions of electron oscillations, thus leading to a larger number of electrons generated and to an increase in their temperature, along with a broader angular distribution.

The electron sheath distribution shortly after the end of laser-target interaction can be extracted from the PIC simulations as shown in Figs. 4(c) and 4(d) for both NST and PT. The shape of the hot electron cloud on the target rear side (electron sheath distribution) is different due to the initial angular distribution of hot electrons generated on the target front side. In fact, after the initial propagation through the target, the electrons recirculate back and forth and also propagate towards the target lateral sides. Our interpretation is that a narrow angular distribution of the generated hot electrons for a plane target leads to electron filamentation in the dielectric foil. However, we are not able to consider such filamentation by numerical simulations, since PIC simulations have to start with the presence of a "warm" plasma having some ionization degree since the very beginning, which is a mandatory assumption due to numerical constraints.

The most interesting feature in our results is the homogeneous beam profile obtained with a nanosphere dielectric target, as already shown in Fig. 3. This homogeneous spatial profile is particularly evident when it is compared to the one obtained with a planar insulator target where typical irregular features are present. According to Refs. [16,26], the observation of irregular structures in the beam profile on RCF is attributed to electron filaments

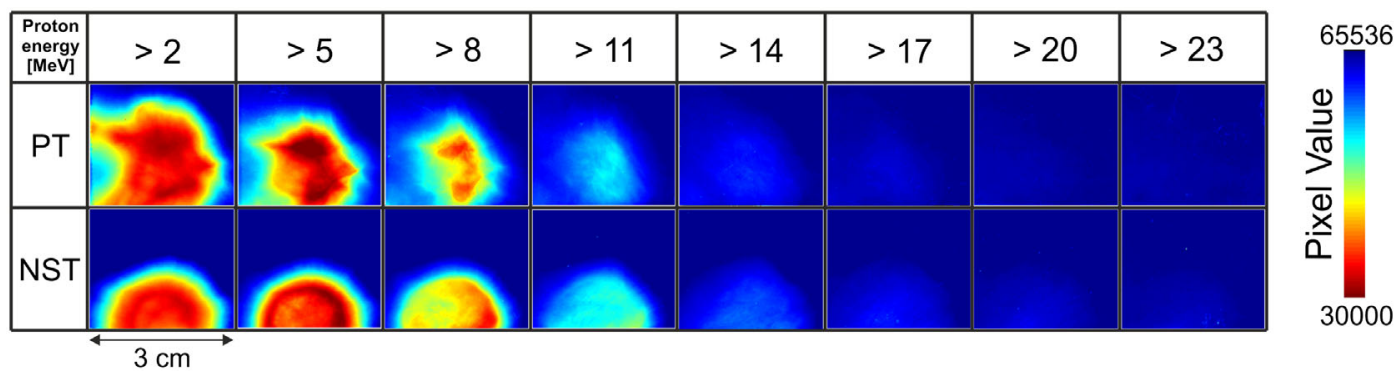

FIG. 3. Proton beam profile using red color channel for PT and NST targets obtained from the RCF diagnostics. 

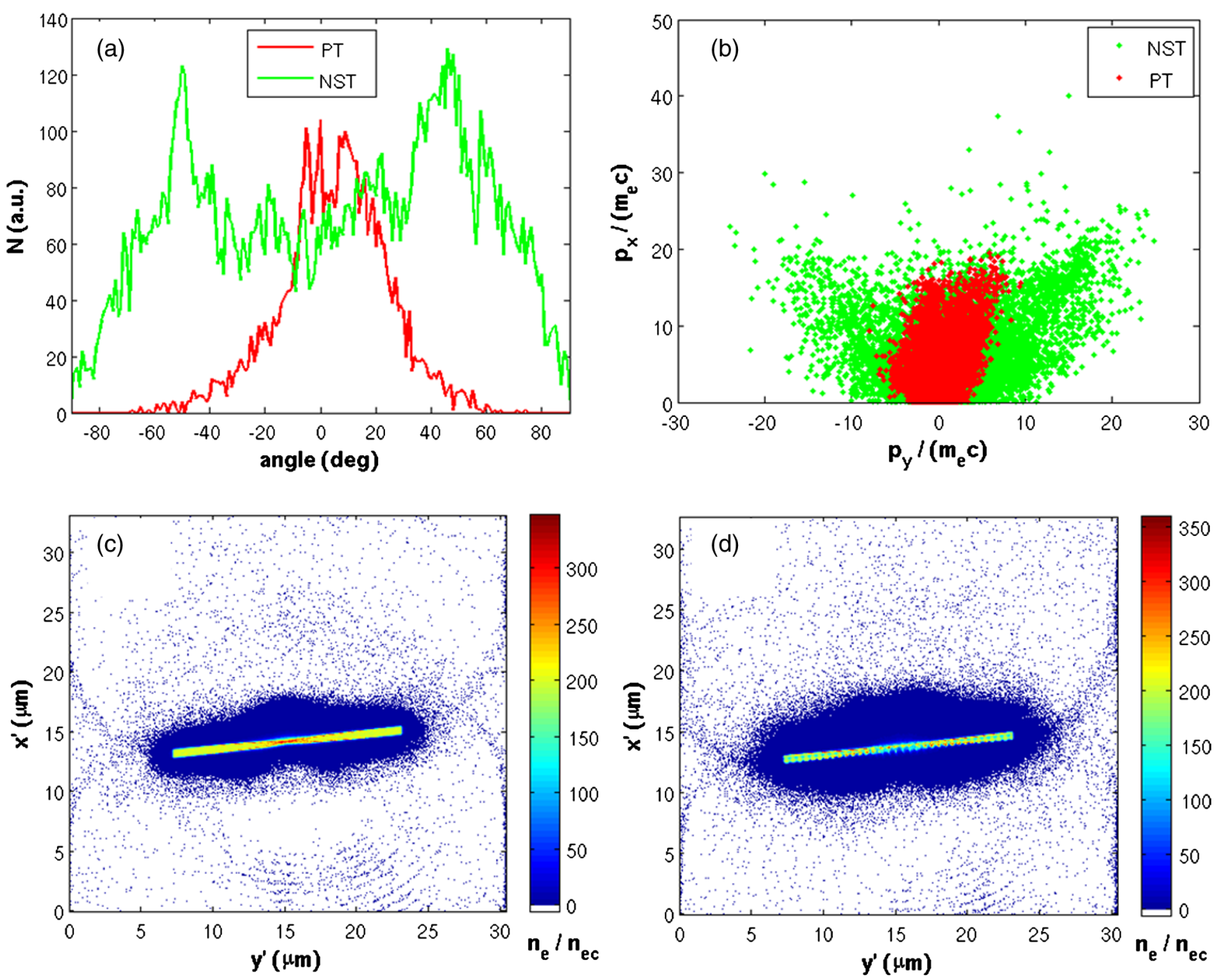

FIG. 4. (a) Angular distribution of hot electrons with energy higher than $1 \mathrm{MeV}$ during laser-target interaction in the forward direction. (b) Momentum space of hot electrons in the forward direction; electrons moving along the target normal direction have a perpendicular component of momentum $\mathrm{p}_{\mathrm{y}}=0$. (c) Hot electron distribution for a PT; (d) hot electron distribution for a NST.

developed in insulators. This filamentation is ascribed to the instability of the ionization front related to the electric field ionization process [26]. Only a small perturbation on an underlying smooth hot electron sheath distribution can lead to inhomogeneity in the spatial profile of the accelerated protons and typically appears for insulator layers thicker than $100 \mathrm{~nm}[16,27]$. On the other hand, a broad (relatively low current density) and homogeneous angular distribution of hot electrons generated in a nanosphere target can mitigate such filamentation, resulting in a homogeneous proton beam profile.

The difference in spatial proton beam profiles between planar and nanosphere targets can be partially explained with the help of PIC simulation results. Figure 5 shows the proton density distributions about $150 \mathrm{fs}$ after the lasertarget interaction. In the case of a planar foil [Fig. 5(a)], a narrow peak structure is observed in the expanding proton front. This narrow ion beam might show irregular structures in the spatial beam profile (as recorded experimentally by $\mathrm{RCFs}$ ) due to filamentation experienced by hot electrons. In fact, even if the electron filamentation cannot be observed directly in our PIC simulations (because ionization and collisions are not included), it is reasonable to assume that the narrow angular distribution of hot electrons (relatively high current density) can generate filamentation when propagating through an insulator target, in agreement with previous theoretical and experimental findings $[16,27,28]$. On the other hand, for a nanosphere target [Fig. 5(b)], a broader proton front profile with two smaller peaks (corresponding to the ring structure observed experimentally) is shown. This might be ascribed to the absence of filamentation in the hot electron population when propagating through the insulator target thanks to their broad and homogeneous spatial distribution. 

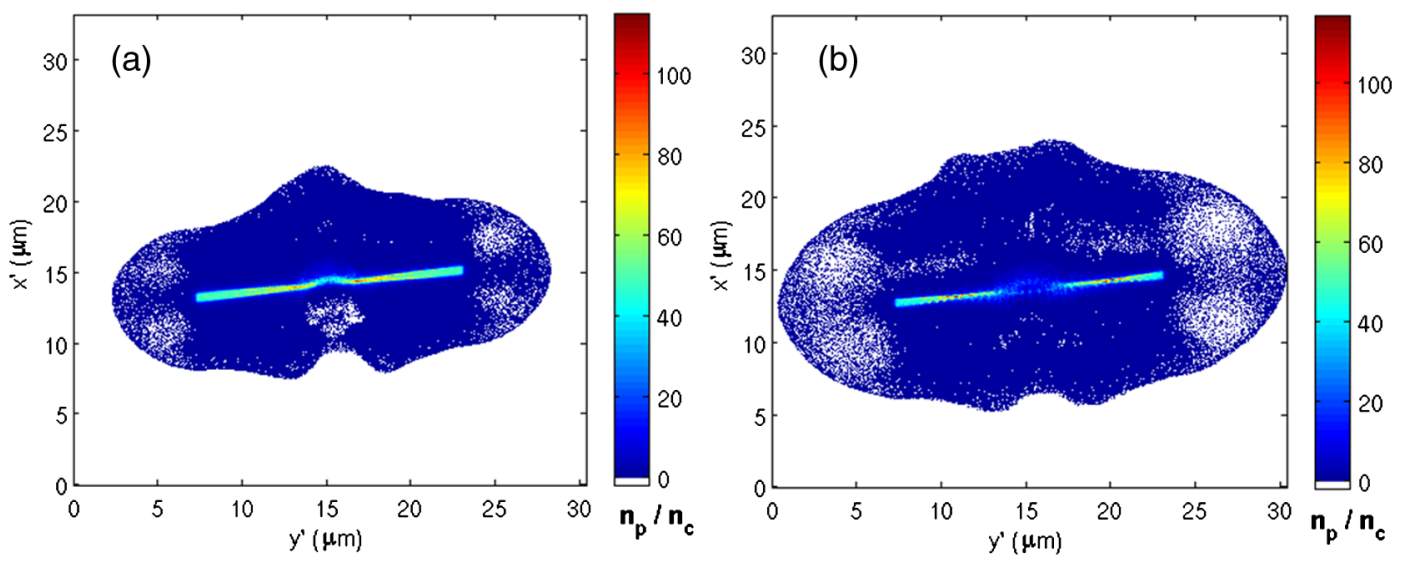

FIG. 5. Proton density distribution after laser-target interaction (a) for a planar target and (b) for a nanosphere target.

\section{CONCLUSIONS}

We have extended the validity of the micron-thick nanosphere targets to higher intensities (up to $7 \times 10^{20} \mathrm{~W} / \mathrm{cm}^{2}$ ) by enhancing the cutoff energy and the number of laserdriven protons with respect to our previous achievements $[14,15]$. Besides, we have shown for the first time that a nanosphere dielectric target could produce a homogeneous beam profile with a constant divergence angle. In fact, insulator targets are widely investigated by research groups working in this field, since they present the main advantage to allow reducing shot-to-shot fluctuations in the accelerated proton beam number compared to metallic foils where the protons are accelerated from hydrocarbon impurities on the target rear side, thus leading to large fluctuations in the proton beam current. On the other hand, dielectric targets typically show an inhomogeneous spatial profile due to filamentation effects of the hot electrons which develop during their propagation through the insulator foil. This is a clear drawback which typically limits the use of such targets, especially when ultrahigh intensities, and hence large hot electron current densities, are used.

The homogeneous proton beam profile could be partially explained by the mitigation of hot electron instabilities due to an increased transverse momentum component. Thus, from both our experimental and numerical results, it can be concluded that homogeneous and high-energy proton beams having high-conversion efficiency can be attained by using nanosphere dielectric targets. A continuous effort to produce homogeneous and high-yield $100-\mathrm{MeV}$-level proton beams from a nanosphere insulator target should be pursued for biomedical applications having a high societal impact. According to our numerical simulations, a $100-\mathrm{MeV}$ proton cutoff energy can be achieved at a laser intensity of about $5 \times 10^{21} \mathrm{~W} / \mathrm{cm}^{2}$ by using a focusing mirror with a shorter focal length and by improving the reflection efficiency of a plasma mirror. Moreover, a clear increase of the proton yield (a factor of 2.5 for NST compared to PT) in an energy range already of interest for medical applications (73-83 MeV) [29] is achievable.
From a practical point of view, a micron-thick nanosphere dielectric target can provide a freestanding, robust, and easy-to-handle target solution, and the improved spatial features obtained with a nanosphere target offer additional benefits in delivering proton beams to a potential user station for applications (such as radiobiology or future laser-based hadron therapy) through a beam transport system using magnetic optics. In fact, the homogeneous beam profile and its constant divergence angle are crucial features in designing the whole beam transport section for applications. In conclusion, due to the increasing demand of reliable proton beams for multidisciplinary applications, the use of nanosphere dielectric targets can be considered as a promising solution to provide high-energy and high-yield proton beam sources with an improved beam quality in terms of the spatial profile.

\section{ACKNOWLEDGMENTS}

The technical supports provided by the Center for Relativistic Laser Science of Institute for Basic Science, Korea, and by the Advanced Photonics Research Institute of GIST, Korea, are greatly acknowledged. This work was supported by IBS (Institute for Basic Science, Korea) under IBS-R012-D1. This research was also supported by the Academy of Sciences of the Czech Republic (M100101210), by the Ministry of Education, Youth and Sports of the Czech Republic (ELI-Beamlines reg. No. CZ.1.05/1.1.00/02.0061 and project No. LD14089), by Czech Science Foundation Project No. 15-02964S, and partially by the project "Centre for Innovation and Technology Transfer" (Project No. CZ.1.05/3.1.00/10.0210).

[1] S. V. Bulanov and V. S. Khoroshkov, Plasma Phys. Rep. 28, 453 (2002).

[2] R. A. Snavely, M. H. Key, S. P. Hatchett, T. E. Cowan, M. Roth, T. W. Phillips, M. A. Stoyer, E. A. Henry, T. C. Sangster, M. S. Singh, S. C. Wilks, A. MacKinnon, 
A. Offenberger, D. M. Pennington, K. Yasuike, A. B. Langdon, B. F. Lasinski, J. Johnson, M. D. Perry, and E. M. Campbell, Phys. Rev. Lett. 85, 2945 (2000); S. A. Gaillard, T. Kluge, K. A. Flippo, M. Bussmann, B. Gall, T. Lockard, M. Geissel, D. T. Offermann, M. Schollmeier, Y. Sentoku, and T.E. Cowan, Phys. Plasmas 18, 056710 (2011).

[3] B. M. Hegelich, B. J. Albright, J. Cobble, K. Flippo, S. Letzring, M. Paffett, H. Ruhl, J. Schreiber, R. K. Schulze, and J. C. Fernandez, Nature (London) 439, 441 (2006).

[4] A. Henig, S. Steinke, M. Schnurer, T. Sokollik, R. Horlein, D. Kiefer, D. Jung, J. Schreiber, B. M. Hegelich, X. Q. Yan, J. Meyer-ter-Vehn, T. Tajima, P. V. Nickles, W. Sandner, and D. Habs, Phys. Rev. Lett. 103, 245003 (2009).

[5] S. C. Wilks, A. B. Langdon, T. E. Cowan, M. Roth, M. Singh, S. Hatchett, M. H. Key, D. Pennington, A. MacKinnon, and R. A. Snavely, Phys. Plasmas 8, 542 (2001).

[6] T. Esirkepov, M. Borghesi, S. V. Bulanov, G. Mourou, and T. Tajima, Phys. Rev. Lett. 92, 175003 (2004).

[7] A. Macchi, F. Cattani, T. V. Liseykina, and F. Cornolti, Phys. Rev. Lett. 94, 165003 (2005).

[8] L. Yin, B. J. Albright, K. J. Bowers, D. Jung, J. C. Fernández, and B. M. Hegelich, Phys. Rev. Lett. 107, 045003 (2011).

[9] D. Jung, L. Yin, B. J. Albright, D. C. Gautier, S. Letzring, B. Dromey, M. Yeung, R. Hörlein, R. Shah, S. Palaniyappan, K. Allinger, J. Schreiber, K. J. Bowers, H. C. Wu, J. C. Fernandez, D. Habs, and B. M. Hegelich, New J. Phys. 15, 023007 (2013).

[10] S. Kar, K. F. Kakolee, B. Qiao, A. Macchi, M. Cerchez, D. Doria, M. Geissler, P. McKenna, D. Neely, J. Osterholz, R. Prasad, K. Quinn, B. Ramakrishna, G. Sarri, O. Willi, X. Q. Yuan, M. Zepf, and M. Borghesi, Phys. Rev. Lett. 109, 185006 (2012).

[11] I J. Kim, K. H. Pae, C. M. Kim, H. T. Kim, J. H. Sung, S. K. Lee, T. J. Yu, I. W. Choi, C.-L. Lee, K. H. Nam, P. V. Nickles, T. M. Jeong, and J. Lee, Phys. Rev. Lett. 111, 165003 (2013).

[12] H. Daido, M. Nishiuchi, and A. S. Pirozhkov, Rep. Prog. Phys. 75, 056401 (2012).

[13] O. Klimo, J. Psikal, J. Limpouch, J. Proska, F. Novotny, T. Ceccotti, V. Floquet, and S. Kawata, New J. Phys. 13, 053028 (2011).

[14] D. Margarone, O. Klimo, I. J. Kim, J. Prokupek, J. Limpouch, T. M. Jeong, T. Mocek, J. Psikal, H. T. Kim, J. Proska, K. H. Nam, L. Stolcova, I. W. Choi, S. K. Lee, J. H. Sung, T. J. Yu, and G. Korn, Phys. Rev. Lett. 109, 234801 (2012).

[15] D. Margarone, O. Klimo, IJ. Kim, J. Prokupek, J. Limpouch, T. M. Jeong, T. Mocek, J. Psikal, H. T. Kim, J. Proska, K. H Nam, I. W. Choi, T. Levato, L. Stolcova, S. K. Lee, M. Krus, F. Novotny, J. H. Sung, J. Kaufman, T. J. Yu, and G. Korn, in Proceedings of the SPIE Conference: High-Power, High-Energy, and HighIntensity Laser Technology, and Research Using Extreme Light: Entering New Frontiers with Petawatt-Class Lasers, edited by J. Hein, G. Korn, and L. O. Silva (SPIE, Bellingham, WA, 2013), Vol. 8780, p. 878023.

[16] J. Fuchs, T. E. Cowan, P. Audebert, H. Ruhl, L. Gremillet, A. Kemp, M. Allen, A. Blazevic, J.-C. Gauthier, M. Geissel, M. Hagelich, S. Karsch, P. Parks, M. Roth, Y. Sentoku, R. Stephens, and E. M. Campbell, Phys. Rev. Lett. 91, 255002 (2003).

[17] T. M. Jeong and J. Lee, Ann. Phys. (Amsterdam) 526, 157 (2014).

[18] K. A. Ivanov, A. V. Brantov, S. I. Kudryashov, S. V. Makarov, D. A. Gozhev, R. V. Volkov, A. A. Ionin, V. Yu. Bychenkov, and A. B. Savelev, Laser Phys. Lett. 12, 046005 (2015).

[19] A. Choukourov, I. Melnichuk, I. Gordeev, O. Kylián, J. Hanuš, J. Kousal, P. Solař, L. Hanyková, J. Brus, D. Slavínská, and H. Biederman, Thin Solid Films 565, 249 (2014).

[20] J. Limpouch, J. Psikal, O. Klimo, J. Vyskocil, J. Proska, F. Novotny, L. Stolcova, and M. Kveton, in Proceedings of the SPIE Conference: High-Power, High-Energy, and High-Intensity Laser Technology and Research Using Extreme Light: Entering New Frontiers with PetawattClass Lasers, edited by J. Hein, G. Korn, and L. O. Silva (SPIE, Bellingham, WA, 2013), Vol. 8780, p. 878027.

[21] D. Margarone, A. Velyhan, L. Torrisi, M. Cutroneo, L. Giuffrida, A. Picciotto, J. Krasa, S. Cavallaro, J. Limpouch, O. Klimo, J. Psikal, J. Proska, and F. Novotny, Appl. Surf. Sci. 272, 132 (2013).

[22] R. Prasad, D. Doria, S. Ter-Avetisyan, P. S. Foster, K. E, Quinn, L. Romagnani, C. M. Brenner, J. S. Green, P. Gallegos, M. J. V. Streeter, D. C. Carroll, O. Tresca, N. Dover, C. A. J. Palmer, J. Schreiber, D. Neely, Z. Najmudin, P. McKenna, M. Zepf, and M. Borghesi, Nucl. Instrum. Methods Phys. Res., Sect. A 623, 712 (2010).

[23] D. Kirby, S. Green, F. Fiorini, D. Parker, L. Romagnani, D. Doria, S. Kar, C. Lewis, M. Borghesi, and H. Palmans, Laser Part. Beams 29, 231 (2011).

[24] J. Pšikal, J. Limpouch, S. Kawata, and A. A. Andreev, Czech. J. Phys. 56, B515 (2006).

[25] V. Floquet, O. Klimo, J. Psikal, A. Velyhan, J. Limpouch, J. Proska, F. Novotny, L. Stolcova, A. Macchi, A. Sgattoni, L. Vassura, L. Labate, F. Baffigi, L. A. Gizzi, Ph. Martin, and T. Ceccotti, J. Appl. Phys. 114, 083305 (2013).

[26] M. Manclossi, J. J. Santos, D. Batani, J. Faure, A. Debayle, V. T. Tikhonchuk, and V. Malka, Phys. Rev. Lett. 96, 125002 (2006).

[27] M. Roth, A. Blazevic, M. Geissel, T. Schlegel, T. E. Cowan, M. Allen, J.-C. Gauthier, P. Audebert, J. Fuchs, J. Meyer-ter-Vehn, M. Hegelich, S. Karsch, and A. Pukhov, Phys. Rev. ST Accel. Beams 5, 061301 (2002).

[28] M. N. Quinn, D. C. Carroll, X. H. Yuan, M. Borghesi, R. J. Clarke, R. G. Evans, J. Fuchs, P. Gallegos, L. Lancia, K. Quinn, A. P. L. Robinson, L. Romagnani, G. Sarri, C. Spindloe, P. A. Wilson, D. Neely, and P. McKenna, Plasma Phys. Controlled Fusion 53, 124012 (2011).

[29] G. Cuttone et al., Eur. Phys. J. Plus 126, 65 (2011). 